\title{
Methicillin-resistant Staphylococcus aureus development in intensive care patients
}

\author{
A case-control study
}

Mohamed A. Ali, MBBS, Ahmad M. Rajab, MBBS, Abdullah M. Al-Khani, MBBS, Saleh Q. Ayash, MBBS, Amjad Chamsi Basha, MBBS, Ahmed Abdelgadir, MBBS, Tawfik M. Rajab, MBBS, Saed Enabi, MBBS, Nazmus Saquib, PhD.

\begin{abstract}

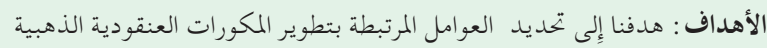

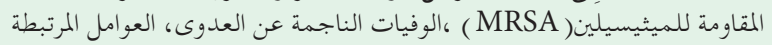
بالوفيات المبكرة و المتأخرة في عينة من مرضى العناية المركزة

الطريقة : كانت الحالات ( العدد = 44 ) من مرضى وحدة العناية المركزة الذين تم

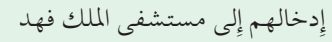

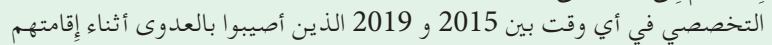

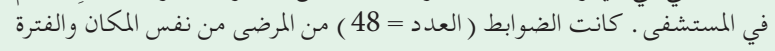

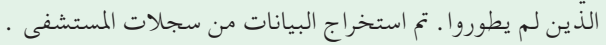

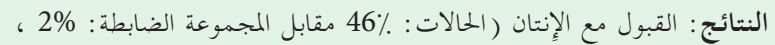

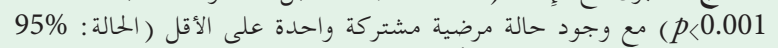

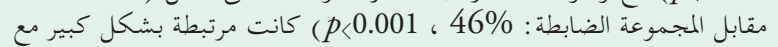

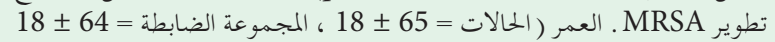
،

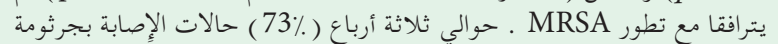

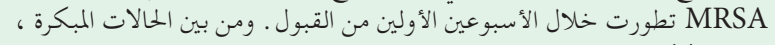

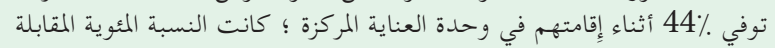

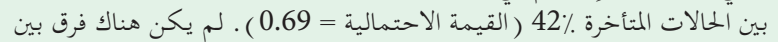

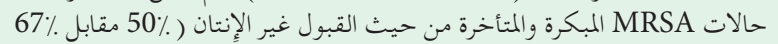

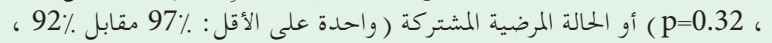
$\cdot(\mathrm{p}=0.17$

الحخلاصة: الإنتان والحالات المرضية المصاحبة كانت عوامل خطر كبيرة لتطور

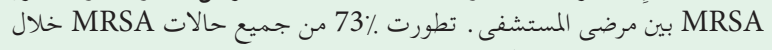
الأسبوعين الأولين من القبول . المبتى .

Objectives: To determine the factors associated with the development of methicillin-resistant Staphylococcus aureus (MRSA), hospital stay and mortality, and early versus late MRSA infection.

Methods: Cases ( $\mathrm{n}=44)$ were intensive care unit (ICU) patients admitted to King Fahd Specialist Hospital, Al-Qassim, Saudi Arabia between 2015 and 2019 who developed MRSA during their hospital stay. Controls $(n=48)$ were patients from the same place and period who did not develop MRSA. Data were abstracted from hospital records.
Results: Admission with sepsis (case: $46 \%$ vs. control: $2 \%, p<0.001)$ and having at least one comorbid condition (case: $95 \%$ vs. control: $46 \%, p<0.001$ ) were significantly associated with the development of MRSA. Age (mean \pm SD: case: $65 \pm 18$, control: $64 \pm 18, p=0.7$ ) and gender ( $\%$ male, case: $52 \%$, control: $56 \%, p=0.70$ ) were not associated with the development of MRSA. Approximately $73 \%$ of all MRSA cases developed within the first 2 weeks of admission. Among the early cases, $44 \%$ died during their ICU stay; the corresponding percentage among the late cases was $42 \% \quad(p=0.69)$. There was no difference between early and late MRSA cases in terms of non-sepsis admissions (50\% vs. $67 \%$, $p=0.32$ ) or comorbid status (at least one: $97 \%$ vs. $92 \%$, $p=0.17)$.

Conclusion: Sepsis and comorbid conditions were significant risk factors for MRSA development among hospital patients.

Keywords: MRSA, Saudi Arabia, ICU, hospital-acquired infection

Saudi Med J 2020; Vol. 41 (11): 1181-1186 doi: 10.15537/smj.2020.11.25465

From the College of Medicine, Sulaiman Al Rajhi University, Al-Qassim, Kingdom of Saudi Arabia.

Received 15th June 2020. Accepted 6th October 2020.

Address correspondence and reprint request to: Dr. Nazmus Saquib, College of Medicine, Sulaiman Al Rajhi University, Al-Qassim, Kingdom of Saudi Arabia.E-mail: a.saquib@sr.edu.sa

ORCID ID: https://orcid.org/0000-0002-2819-2839 
$\mathrm{P}$ atients in the hospital, particularly those in the intensive care unit (ICU), are at increased risk of contracting hospital-acquired infections. ${ }^{1}$ In addition to Escherichia coli, Klebsiella, Pseudomonas and Acinetobacter, Staphylococcus aureus (S. aureus) is a common organism responsible for such infections. Staphylococcus aureus is often resistant to commonly used antibiotics, such as methicillin (a B-lactam of the penicillin class), amoxicillin, and penicillin. ${ }^{1}$ Methicillin-resistant Staphylococcus aureus (MRSA) commonly affects skin or soft-tissues and often occurs with other co-infections, such as a methicillin-resistant Pseudomonas infection. ${ }^{2}$ The majority of MRSA studies in Saudi Arabia have focused on epidemiological trends, including its prevalence and incidence among patients and healthcare workers, its source (for example, nosocomial or community-acquired), its various strains, and its response to antibiotics. ${ }^{3-11}$ Reported MRSA cases were highest in Riyadh (central) and Asir (southwest) regions, followed by Makkah and other cities in the western regions. ${ }^{3,6,7}$ The western city of Jeddah reported a prevalence as high as 38\% among hospital patients. ${ }^{3}$ The majority of studies have reported a higher prevalence of MRSA in males than in females, 7 although contrary evidence does exist. ${ }^{4} \mathrm{~A}$ study from the neighboring United Arab Emirates identified female gender as a risk factor for incident MRSA among ambulatory patients who came to the hospital with a skin or soft tissue infection. Methicillin-resistant Staphylococcus aureus is more commonly acquired during hospital stay than from community living. ${ }^{12}$ Of the hospitalacquired MRSA, around half of the cases are actual infections, and the rest are colonization. ${ }^{13}$ Saudi Arabia and neighboring Gulf countries have observed a wide variety of strains among MRSA cases. ${ }^{5,10,11}$ The Muslim pilgrimages to Makkah and Medina might contribute to the propagation of diverse MRSA strains, as there is evidence of MRSA's high transmissibility through the respiratory route. ${ }^{14}$ In fact, MRSA has been shown to be one of the main organisms that affects the pilgrims and causes them pneumonia. ${ }^{15}$ Though there is an abundance of MRSA studies on the disease itself in Saudi Arabia, there is limited evidence on patient outcomes. ${ }^{16-18}$ Available studies chose mortality as a patient outcome. The mortality was found to be high, but the rate varied among the studies. ${ }^{3,9}$ For example,

Disclosure. Authors have no conflict of interests, and the work was not supported or funded by any drug company. in a study in Jeddah, $61 \%$ of MRSA patients died, $38 \%$ of whom died from their infection. ${ }^{3}$ Other patient outcomes such as prognostic factors or hospital stay were not evaluated. Studies from elsewhere in the world have reported older age, the severity of patient condition, longer hospital stay, and immunosuppression as risk factors associated with MRSA. ${ }^{17}$ Studies have shown that ICU patients who are MRSA-positive are more likely to develop a systemic MRSA infection and to have a longer hospital stay compared to patients who are MRSA-negative. ${ }^{18}$ For example, in Glasgow, United Kingdom, the development of MRSA among ICU patients significantly increased their length of hospital stay though it did not increase their risk of death. ${ }^{16}$

All available Saudi studies on MRSA are patientonly studies; in the absence of an appropriate control group, it is not possible to assess the factors related to the development and prognosis of MRSA. Hence, with a case-control design, the current study aimed to assess i) the factors related to the development of MRSA, ii) hospital stay and its association with mortality, and iii) the factors associated with early versus late MRSA development.

Methods. We conducted a case-control study in King Fahad Specialist Hospital (KFSH), Al-Qassim, Saudi Arabia. The Al-Qassim regional research ethics committee (Ministry of Health, approval number 20181118) reviewed and approved the study protocol.

Case definition are all patients who were $\geq 18$ years of age admitted to the ICU of KFSH between January 2015 and November 2019 and were diagnosed with MRSA. They screened negative for MRSA at the beginning of their admission to the ICU. They developed MRSA thereafter, during their stay in the unit. A positive nasal swab $(n=20)$, blood culture $(n=12)$, wound culture $(n=7)$, urine culture, or sputum culture $(n=5)$ was used to confirm the diagnosis of MRSA $(n=44)$. Pregnant patients were excluded.

Control definition are patients who were $\geq 18$ years of age admitted to the ICU of KFSH anytime between January 2015 and November 2019 and did not develop MRSA during their ICU stay $(n=48)$. Controls were matched with cases by age ( \pm 2 years).

We reviewed the medical records of cases and controls and extracted the following data: age, gender, nationality, admission diagnosis, admission date, MRSA culture date, duration to develop MRSA for cases (time to MRSA in days), antibiotic used for the treatment of MRSA, sepsis status (sepsis or septic shock, non-septic), comorbid conditions (chronic kidney injury, hypertension, diabetes mellitus), and outcome at 
discharge status (alive, deceased). We used the Charlson comorbidity index to identify patients' comorbidities. ${ }^{19}$

Statistical analysis. We entered the extracted data into the IBM SPSS Statistics for Mac, version 24.0 (IBM Corp., Armonk, N.Y., USA). Descriptive statistics were reported with mean and standard deviation (SD) for continuous variables and frequency of figures and tables for categorical variables. We graphed the frequency of cases by time to develop MRSA (in weeks). Cases were defined as 'early infection' (within 2 weeks) and 'late infection' (after 2 weeks) based on the time it took them to develop MRSA.

We compared cases and controls by age, gender, admission status (septic, not septic), comorbid status (none, one condition, 2 or more conditions), antibiotic choice (vancomycin, linezolid), and deceased (yes, no). We also compared early and late cases by age, gender, admission status (septic, not septic), comorbid status (none, one condition, 2 or more conditions), antibiotic choice (vancomycin, linezolid), and deceased (yes, no).

Results. Between January 2015 and November 2019, 117 patients developed MRSA infection during their stay at KFSH. However, only 44 cases developed MRSA after their admission to the ICU and fulfilled the definition of cases to be included in the analyses. We identified 48 controls during the study period.

Comparison of cases and controls. The mean ages of the cases and controls were 65 years (SD: 18, range: 47-83 years) and 64 years (SD: 18 , range: $46-82$ years). Males comprised $52 \%$ of the cases and $56 \%$ of the controls. The cases and controls did not statistically differ in age or gender. The frequency of non-septic admissions (55\% vs. $98 \%, p<0.001)$ and of having at least one comorbid condition ( $96 \%$ vs. $46 \%, p<0.001$ ) were significantly higher among the cases than controls. The majority of cases $(57 \%)$ received vancomycin, while $43 \%$ received linezolid for treatment of MRSA. Among the cases, $43 \%$ died during their ICU stay; the corresponding percentage among the controls was 29\% $(p=0.214)$. Most cases and controls were non-septic on admission (Table 1). The distribution of comorbid conditions in our sample was as follows: diabetes (cases: 17, controls: 18), hypertension (cases: 20, controls: 18), chronic kidney disease (cases: 10 , controls: 2 ), and miscellaneous conditions (cases: 25, controls: 8).

Time to develop MRSA. Time to MRSA was the difference between the date of admission to ICU and the date of the first MRSA-positive culture. No case developed MRSA before the third day of ICU admission. Most of the cases were diagnosed with MRSA within the first 2 weeks. Early cases (first 14 days) developed during the first week $(\mathrm{n}=17,39 \%)$ and second week $(n=15,34 \%)$. Late cases developed during the third week $(n=3,7 \%)$, the fourth week $(n=4,9 \%)$, the fifth week $(n=3,7 \%)$, and sixth week $(n=2,5 \%)$ (Figure 1). The largest proportion of cases (15\%) started on the third day after ICU admission, followed by $11 \%$

Table 1 - Descriptive comparison of Methicillin-resistant Staphylococcus aureus (MRSA) cases and controls.

\begin{tabular}{lccc}
\hline Variables & Cases $(\mathbf{n}=44)$ & Control $(\mathbf{n}=48)$ & $P$-value \\
\hline $\begin{array}{l}\text { Age (years) (mean } \pm \text { SD) } \\
\text { Gender }\end{array}$ & $65 \pm 18$ & $64 \pm 18$ & 0.748 \\
Male & $23(52.3)$ & $27(56.3)$ & 0.702 \\
Female & $21(47.7)$ & $21(43.8)$ & \\
Admission status & & & \\
Not septic & $24(54.5)$ & $47(97.9)$ & $<0.001$ \\
Sepsis & $20(45.5)$ & $1(2.1)$ & \\
Comorbid status & & & \\
None & $2(4.5)$ & $26(54.2)$ & $<0.001$ \\
1 condition & $21(47.7)$ & $4(8.3)$ & \\
$\geq 2$ conditions & $21(47.7)$ & $18(37.5)$ & \\
Antibiotic Choice & & & Not \\
Vancomycin & $25(56.8)$ & $0(0)$ & applicable \\
Linezolid & $19(43.2)$ & 0.214 \\
Deceased & & & \\
Yes & $19(43.0)$ & $14(29.0)$ & \\
No & $25(57.0)$ & $34(71.0)$ & \\
\hline & Values are presented as number and percentage (\%). & \\
\hline
\end{tabular}

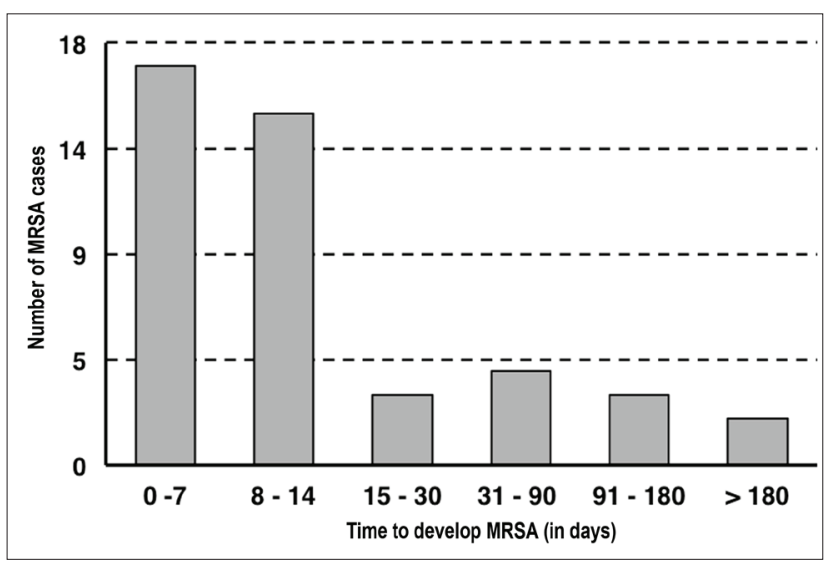

Figure 1-Bar graph of Methicillin-resistant Staphylococcus aureus (MRSA) development in the intensive care unit. 
on the sixth day; approximately $23 \%$ of all cases were positive for the infection within the first 5 days of ICU admission (data not shown).

Comparison between early and late MRSA cases. The mean age of early cases was 63 years (SD: 18, range: 45-81 years); the mean age of late cases was 70 years (SD: 19 , range: 51-89 years). Of the early cases, $59 \%$ were male. Of the late cases, $67 \%$ were female. There was no difference between the early and late MRSA cases in terms of non-sepsis admissions ( $50 \%$ vs. $67 \%, p=0.32$ ) or comorbid status (at least one: $97 \%$ vs. $92 \%, p=0.17$ ). The majority of early and late cases $(56 \%, 58 \%)$ received vancomycin, followed by linezolid $(44 \%, 42 \%)$ for the treatment of MRSA. Among the early cases, 44\% died during their ICU stay; the corresponding percentage among the late cases was $42 \%(p=0.69)$ (Table 2).

Discussion. We explored factors associated with the development of MRSA among ICU patients as well as factors associated with early versus late MRSA infection. The salient findings of this study were the median time for developing MRSA was 10 days, the majority $(73 \%)$ of MRSA cases developed within the first 2 weeks of admission, and 38.6\% developed it in the first week. Patients who had $\geq 2$ comorbid conditions were more likely (56\%) to develop an early infection ( $\leq 2$ weeks after admission), females were more likely than males $(67 \%$ vs. $33 \%)$ to develop a late infection, and deaths were higher in females $(\mathrm{n}=3)$ as compared to males $(n=2)$.

Studies in Saudi Arabia have focused on epidemiological trends of MRSA. For instance, Khanfar et $\mathrm{al}^{5}$ discussed the prevalence, incidence, and possible predictors of MRSA infection, while Eed et $\mathrm{al}^{20}$ discussed the molecular characteristics of MRSA among hospital isolates. Our study is the first in the region to address the in-hospital features of MRSA patients compared to controls. Internationally, though, there have been several case-control studies studying MRSA and its risk factors. When it comes to age group, patients with MRSA were younger (median, 61 vs. 70 years) than matched controls in a Danish study. ${ }^{21}$ In our study, the age difference between cases and controls was less tangible (65 in cases vs. 64 in controls). More consistent with our findings were those of a 2004 study of a similar design which reported the mean age among cases to be $68.2 \pm 15.6$ and $64.9 \pm 19.9$ among controls. ${ }^{22}$

The death rate in our study among MRSA cases was higher $(43 \%)$ compared with the Spanish study $(29.5 \%) .{ }^{23}$ In Jeddah, the total mortality rate of MRSA
Table 2 - Comparing early and late Methicillin-resistant Staphylococcus aureus (MRSA) cases $(\mathrm{n}=44)$.

\begin{tabular}{lccc}
\hline Variables & \multicolumn{2}{c}{ Time of infection } & $P$-value \\
& $\begin{array}{c}\text { Early }(\leq 2 \text { weeks }) \\
(\mathbf{n}=32)\end{array}$ & $\begin{array}{c}\text { Late }(>2 \text { weeks }) \\
(\mathbf{n}=12)\end{array}$ & \\
\hline Age (years) (mean \pm SD) & $63 \pm 18$ & $70 \pm 19$ & 0.244 \\
Gender & & & \\
Male & $19(59.4)$ & $4(33.3)$ & 0.124 \\
Female & $13(40.6)$ & $8(66.7)$ & \\
Admission status & & & \\
Not septic & $16(50.0)$ & $8(66.7)$ & 0.323 \\
Sepsis & $16(50.0)$ & $4(33.3)$ & \\
Comorbid status & & & \\
None & $1(3.1)$ & $1(8.3)$ & 0.170 \\
1 condition & $13(40.6)$ & $8(66.7)$ & \\
$\geq 2$ conditions & $18(56.3)$ & $3(25.0)$ & \\
Antibiotic choice & & & 0.901 \\
Vancomycin & $18(56.3)$ & $7(58.3)$ & \\
Linezolid & $14(43.8)$ & $5(41.7)$ & \\
Deceased & $14(43.8)$ & $5(41.7)$ & 0.698 \\
\hline$\quad$ Values are presented as number and percentage (\%). & \\
\hline
\end{tabular}

patients was $61 \%$, of which only $38 \%$ were due to MRSA infection. ${ }^{3}$ Other studies in Saudi Arabia showed a 15\% mortality in MRSA patients. ${ }^{9}$ The risk of developing MRSA is mainly related to an ICU stay of more than 4 days. ${ }^{2}$ This is in line with our findings, where no MRSA cases developed in the first 3 days of ICU admission. It has been shown that the detection of MRSA doubles within the first and second week of ICU admission. ${ }^{24}$ In our study, the majority of infections were detected within the first 2 weeks of ICU admission. Patients who were MRSA-positive after ICU admission were known to have lengthier hospital stays than MRSA-negative patients matched by age. ${ }^{16}$ Moreover, the risk of MRSA acquisition in ICU patients increases when patients have a confirmed non-MRSA infection within the first 2 days of ICU admission. ${ }^{16}$ Interestingly, results from a meta-analysis of risk factors associated with MRSA colonization showed that admission to an ICU was not associated with increased risk of MRSA colonization. ${ }^{25}$ However, exposure to nosocomial pathogens and prior hospitalization were associated with MRSA carriage, and the type of ICU admission, medical versus surgical, did have a significant association with MRSA carriage. ${ }^{25}$

While comorbid conditions have been associated with MRSA carriage, ${ }^{18}$ McMaster et al $^{16}$ reported 
that MRSA in critically diseased patients did not significantly affect their mortality. In our sample, more males contracted MRSA infection than females. In addition, we found that patients who developed an early infection were more likely to be younger males with more than one comorbidity, while those who developed a late infection were older females with one comorbid condition. In 2013, a prospective study was conducted in Riyadh, Saudi Arabia that revealed a significantly higher prevalence rate in males $(48 \%)$ than in females $(32 \%){ }^{7}$ We reported that patients with multiple comorbidities were more susceptible to contracting a MRSA infection within the first 14 days of ICU admission. One postulation lies in privacy curtains; one study reported that curtains become contaminated within 3 days of being hung, with MRSA positivity increasing 10 to 14 days after curtains are hung around burn units. $^{26}$

Study limitations. The study's case-control design is inherently inferior to other designs. However, due to the rarity of the studied condition and the data available, a case-control study was the only feasible option. The case number was small. The case to control ratio was $1: 1$. Therefore, we did not have much statistical power to detect the difference between cases and controls or between early and late cases. We abstracted the data from medical records, but they did not have comprehensive information, which we could have used in our analysis. For example, the medical records stated that each patient tested negative for MRSA prior to admission to the ICU, but they did not specify the dates of screening. In addition, the full potential of the control group was not reached due to the lack of essential documented data (namely, time until discharge). If designed prospectively, we could investigate other factors that might impact the temporal effect of ICU stays and MRSA infection. A superior study design (namely, retrospective or prospective cohort) would better clarify this relationship.

In conclusion, the first 2 weeks after ICU admission are critical as most MRSA infections were acquired during that period, and more than a third occurred within the first 7 days. Patients with 2 or more comorbid conditions were more likely to develop an early infection. Females were more likely than males to develop a late infection, and deaths were higher in females as compared to males. Optimal care should be provided to all patients along with MRSA-specific preventive measures, especially during their first 2 weeks of ICU admission. Future studies should explore MRSA development in ICU patients in more depth.

\section{References}

1. Thuy DB, Campbell J, Nhat LTH, Hoang NVM, Hao NV, Baker $S$ et al. Hospital-acquired colonization and infections in a Vietnamese intensive care unit. PloS One 2018; 13: e0203600.

2. Ochotorena E, Hernandez Morante JJ, Canavate R, Villegas RA, Viedma I. Methicillin-resistant Staphylococcus aureus and other multidrug-resistant colonizations/infections in an intensive care unit: predictive factors. Biol Res Nurs 2019; 21: 190-197.

3. Madani TA. Epidemiology and clinical features of methicillinresistant Staphylococcus aureus in the University Hospital, Jeddah, Saudi Arabia. Can J Infect Dis Med 2002; 13: 245-250.

4. Ahmad S. The prevalence of Staphylococcus aureus colonization among healthcare workers at a specialist hospital in Saudi Arabia. J Clin Diagn Res 2010; 4: 2438-2441.

5. Khanfar H, Senok A, Anani A, Zinkevich V. Methicillinresistant Staphylococcus aureus transmission in a low-prevalence healthcare setting. J Infect Public Heal 2012; 5: 311-336.

6. Al Yousef SA, Mahmoud SY, Eihab MT. Prevalence of methicilin-resistant Staphylococcus aureus in Saudi Arabia: systemic review and meta-analysis. African Journal of Clinical and Experimental Microbiology 2013; 14: 146-154.

7. Al-Humaidan OS, El-Kersh TA, Al-Akeel RA. Risk factors of nasal carriage of Staphylococcus aureus and methicillin-resistant Staphylococcus aureus among health care staff in a teaching hospital in central Saudi Arabia. Saudi Med J 2015; 36: 1084-1090.

8. Zakai SA. Prevalence of methicillin-resistant Staphylococcus aureus nasal colonization among medical students in Jeddah, Saudi Arabia. Saudi Med J 2015; 36: 807.

9. Alrabiah K, Al Alola S, Al Banyan E, Al Shaalan M, Al Johani S. Characteristics and risk factors of hospital acquired - methicillinresistant Staphylococcus aureus (HA-MRSA) infection of pediatric patients in a tertiary care hospital in Riyadh, Saudi Arabia. Int J Pediatr Adolesc Med 2016; 3: 71-77.

10. Senok A, Ehricht R, Monecke S, Al-Saedan R, Somily A. Molecular characterization of methicillin-resistant Staphylococcus aureus in nosocomial infections in a tertiary-care facility: emergence of new clonal complexes in Saudi Arabia. New Microbes and New Infections 2016; 14: 13-18.

11. Senok A, Somily A, Raji A, Gawlik D, Al-Shahrani F, Baqi S et al. Diversity of methicillin-resistant Staphylococcus aureus CC22-MRSA-IV from Saudi Arabia and the Gulf region. Int J Infect Dis 2016; 51: 31-35

12. El Amin NM, Faidah HS. Methicillin-resistant Staphylococcus aureus in the western region of Saudi Arabia: prevalence and antibiotic susceptibility pattern. Ann Saudi Med 2012; 32: 513-516.

13. Balkhy HH, Memish ZA, Almuneef MA, Cunningham GC, Francis C, Fong KC et al. Methicillin-resistant Staphylococcus aureus: a 5-year review of surveillance data in a tertiary care hospital in Saudi Arabia. Infect Control Hosp Epidemiol 2007; 28: 976-982.

14. Al-Zahrani IA, Azhar EI, Jiman-Fatani AA, Siddig LA, Yasir M, $\mathrm{Al}-\mathrm{Ghamdi} \mathrm{AK}$ et al. Impact of mass migrations on the clonal variation of clinical Staphylococcus aureus strains isolated from the Western region of Saudi Arabia. J Infect Public Heal 2019; 12: 317-322.

15. Shirah BH, Zafar SH, Alferaidi OA, Sabir AMM. Mass gathering medicine (Hajj Pilgrimage in Saudi Arabia): the clinical pattern of pneumonia among pilgrims during Hajj. $J$ Infect Public Heal 2017; 10: 277-286. 
16. McMaster J, Booth MG, Smith A, Hamilton K. Meticillinresistant Staphylococcus aureus in the intensive care unit: its effect on outcome and risk factors for acquisition.J Hosp Infect 2015; 90: 327-332.

17. Callejo-Torre F, Eiros Bouza JM, Olaechea Astigarraga P, Coma Del Corral MJ, Palomar Martinez M, Alvarez-Lerma F et al. Risk factors for methicillin-resistant Staphylococcus aureus colonisation or infection in intensive care units and their reliability for predicting MRSA on ICU admission. Infez Med 2016; 24: 201-209.

18. Qiao F, Huang W, Cai L, Zong Z, Yin W. Methicillin-resistant Staphylococcus aureus nasal colonization and infection in an intensive care unit of a university hospital in China. J Int Med Res 2018; 46: 3698-3708.

19. Charlson ME, Pompei P, Ales KL, MacKenzie CR. A new method of classifying prognostic comorbidity in longitudinal studies: development and validation. J Chronic Dis 1987; 40: 373-383.

20. Eed EM, Ghonaim MM, Hussein YM, Saber TM, Khalifa AS. Phenotypic and molecular characterization of HA-MRSA in Taif hospitals, Saudi Arabia. J Infect Dev Ctries 2015; 9: 298-303.

21. Jensen AG, Wachmann CH, Poulsen KB, Espersen F, Scheibel J, Skinhøj P et al. Risk factors for hospital-acquired Staphylococcus aureus bacteremia. Arch Intern Med 1999; 159: 1437-1444.
22. Tacconelli E, Venkataraman L, De Girolami PC, Dagata EMC. Methicillin-resistant Staphylococcus aureus bacteraemia diagnosed at hospital admission: distinguishing between community-acquired versus healthcare-associated strains. $J$ Antimicrob Chemother 2004; 53: 474-479.

23. Pujol M, Peña C, Pallares R, Ayats J, Ariza J, Gudiol F. Risk factors for nosocomial bacteremia due to methicillin-resistant Staphylococcus aureus. Eur J Clin Microbiol Infect Dis 1994; 13: 96-102.

24. Durmaz G, Sanci O, Oz Y, Guven K, Kiremitci A, Aksit F. Methicillin-resistant $S$. aureus colonization in intensive care unit patients: early identification and molecular typing. J Infect Dev Ctries 2016; 10: 465-471.

25. McKinnell JA, Miller LG, Eells SJ, Cui E, Huang SS. A systematic literature review and meta-analysis of factors associated with methicillin-resistant Staphylococcus aureus colonization at time of hospital or intensive care unit admission. Infect Control Hosp Epidemiol. 2013; 34: 1077-1086.

26. Shek K, Patidar R, Kohja Z, Liu S, Gawaziuk JP, Gawthrop M, et al. Rate of contamination of hospital privacy curtains in a burns/plastic ward: a longitudinal study. Am J Infect Control 2018; 46: 1019-1021. 